\title{
Transport process of ions in insulating media in the hyperbolic diffusion regime
}

\author{
G. Barbero ${ }^{1}$ and J. Ross Macdonald ${ }^{2}$ \\ ${ }^{1}$ Dipartimento di Fisica and C.N.I.S.M., Politecnico di Torino, Corso Duca degli Abruzzi 24, 10129 Torino, Italy \\ ${ }^{2}$ Department of Physics and Astronomy, University of North Carolina, Chapel Hill, North Carolina 27599, USA
}

(Received 9 February 2010; revised manuscript received 16 March 2010; published 5 May 2010)

\begin{abstract}
We extend the microscopic Poisson-Nernst-Planck theory of the effects of mobile charge carriers, completely blocked at the electrodes, on the properties of dielectric materials by incorporating a finite speed of response propagation. The usual microscopic theory is based on the assumption that the diffusion current is given by Fick's law, relating the current density with the gradient of concentration at the same time. On the contrary we assume that the flux of diffusing particles is delayed with respect to the concentration gradient, as suggested by extended thermodynamics formulations. We show that, in the hyperbolic diffusion regime approximation, new trends for the real and imaginary parts of the small-signal electrical impedance of a dielectric containing ions versus the frequency of the applied voltage are expected when the delay time is comparable with the Debye relaxation time. In particular, at sufficiently high frequencies the real part of the conductivity, normally independent of frequency, decreases toward zero because of the finite, rather than the infinite propagation speed present in the hyperbolic diffusion regime model.
\end{abstract}

DOI: 10.1103/PhysRevE.81.051503

PACS number(s): 82.45.-h, 77.22.- d, 66.10.Ed

\section{INTRODUCTION}

The fundamental equations describing the redistribution of ions in a dielectric material upon the application of an external electric field were discussed in the continuum approximation long ago by Macdonald [1] and later extended in Refs. [2,3]. They are the continuity equations for the positive and negative charge carriers and Poisson's equation for the electric field across the cell. This work included such effects as generation recombination of the charge carriers, their possibly different valences and mobilities, and involved electrode boundary conditions allowing reactions there. It therefore represents application of the Poisson-Nernst-Planck (PNP) equations to that situation. In Refs. [4,5] simplified conditions were considered, particularly for completely blocking electrodes, the situation considered herein.

In [1-5] the ionic density of currents contains a diffusion component, due to the nonhomogeneity of the ionic density, and a drift term, related to the electric field. The diffusion components of the density of current have been written by assuming the validity of the law of Fick relating the current density to the gradient of concentration at the same time [6]. The PNP equations determine the time evolution of the local bulk density of ions generated by decomposition of impurities and molecular dissociation present in the dielectric material, taking into account the recombination phenomenon when the sample is subjected to an external field. The actual electric field in the liquid is deduced by solving Poisson's equation relating the divergence of the electric field to the net ionic charge.

In the general case where the mobility of the positive ions is different from that of the negative ions, the PNP approach has been used to analyze several situations of practical interest with different electrode boundary conditions [3,7-10]. In particular, these equations have been employed to investigate the impedance spectroscopy of an electrolytic cell with blocking electrodes $[4,5]$. The analysis presented in [5] is valid for arbitrary mobilities, arbitrary valence numbers, and any degree of charge dissociation. The analysis has also been extended to the case in which more than one group of ions is present in the insulating liquid [2,10-12]. Our present aim is to extend the theory to the constrained diffusion case where the mobilities of the ions are hindered by the internal structure of the medium [13-17]. We base the analysis on extended thermodynamics, where the diffusion current at the time $t$ depends on the gradient of concentration at the time $t-\tau$, where $\tau$ is a phenomenological parameter of the model [18]. Preliminary investigations on the same subject have been reported in [19-21].

It is important to note that all of the standard PNP analyzes approaches involve an infinite velocity of propagation on the application of a disturbance, resulting in the immediate presence of nonzero diffusing charges everywhere throughout the material [18]. Such nonphysical diffusion behavior, noted even by Maxwell in 1867 [22], is avoided in the hyperbolic diffusion regime model $[23,24]$. No earlier analyzes involved the satisfaction of the Poisson equation, however, as in the present work.

Our paper is organized as follows. In Sec. II we summarize the standard derivation of the impedance of a cell involving two plane-parallel blocking electrodes, one that employs Fick's law $[3,4]$. The extension of the model to the case where the diffusion current is delayed with respect to the gradient of concentration is discussed in Sec. III. The numerical analysis of the impedance spectroscopy of a cell of an insulating material such as a dielectric liquid containing ions is reported in Sec. IV.

\section{STANDARD ANALYSIS}

According to the law of Fick, if the bulk distribution of particles, $n$, is not homogeneous, a net density of current, $\mathbf{j}$, exists. The equation relating $\mathbf{j}$ with the spatial inhomogeneity of $n$ is

$$
\mathbf{j}(\mathbf{r}, t)=-D \nabla n(\mathbf{r}, t),
$$

where $D$ is the diffusion coefficient. This equation states that the current density $\mathbf{j}(\mathbf{r}, t)$ at the point $\mathbf{r}$ and time $t$ is due to 
the gradient of the bulk density $n(\mathbf{r}, t)$, at the same time $t$. By substituting Eq. (1) in the equation of continuity

$$
\frac{\partial n(\mathbf{r}, t)}{\partial t}=-\nabla \cdot \mathbf{j}(\mathbf{r}, t)
$$

stating the conservation of the number of particles, we get

$$
\frac{\partial n(\mathbf{r}, t)}{\partial t}=D \nabla^{2} n(\mathbf{r}, t)
$$

which is the diffusion equation. It has to be solved with the boundary condition

$$
\mathbf{k} \cdot \mathbf{j}(\mathbf{r}, t)=0,
$$

where $\mathbf{k}$ is the geometrical normal of the surface $S$ limiting the sample, outward directed. Equation (4) holds in the case where the particles cannot leave the sample. Equation (3) with boundary condition Eq. (4) has to be solved with the initial boundary condition $n(\mathbf{r}, 0)=n_{0}(\mathbf{r})$ [6]. For an unbounded space the solution of Eq. (3), in an isotropic medium, is [25]

$$
n(\mathbf{r}, t)=\frac{1}{(4 \pi D t)^{3 / 2}} \int_{V_{\infty}} d \mathbf{u} n_{0}(\mathbf{u}) \exp \left\{-\frac{(\mathbf{r}-\mathbf{u})^{2}}{4 D t}\right\},
$$

where $d \mathbf{u}=d u_{x} d u_{y} d u_{z}$ and the integration on $V_{\infty}$ means on $-\infty \leq u_{x} \leq \infty,-\infty \leq u_{y} \leq \infty,-\infty \leq u_{z} \leq \infty$.

If the particles under investigation are electrically charged, the problem has to be solved by taking into account their contribution to the actual electric field in the sample [1]. We indicate by $n_{p}$ and $n_{m}$ the bulk densities of positive and negative ions. In this case the bulk density of currents is

$$
\begin{gathered}
\mathbf{j}_{p}(\mathbf{r}, t)=-D_{p} \nabla n_{p}(\mathbf{r}, t)+\mu_{p} n_{p}(\mathbf{r}, t) \mathbf{E}(\mathbf{r}, t), \\
\mathbf{j}_{m}(\mathbf{r}, t)=-D_{m} \nabla n_{m}(\mathbf{r}, t)-\mu_{m} n_{m}(\mathbf{r}, t) \mathbf{E}(\mathbf{r}, t),
\end{gathered}
$$

where $D_{p}, D_{m}, \mu_{p}$, and $\mu_{m}$ are the diffusion coefficients and mobilities of the positive and negative ions, respectively. In Eq. (6) $\mathbf{E}(\mathbf{r}, t)$ is the actual electric field due to the external power supplies and to the charge separation induced by the field itself. By taking into account the conservation of the positive and negative ions we have now the equations of continuity

$$
\begin{gathered}
\frac{\partial n_{p}(\mathbf{r}, t)}{\partial t}=-\boldsymbol{\nabla} \cdot \mathbf{j}_{p}(\mathbf{r}, t), \\
\frac{\partial n_{m}(\mathbf{r}, t)}{\partial t}=-\nabla \cdot \mathbf{j}_{m}(\mathbf{r}, t) .
\end{gathered}
$$

By substituting Eq. (6) into Eq. (7) we get

$$
\frac{\partial n_{p}(\mathbf{r}, t)}{\partial t}=D_{p} \boldsymbol{\nabla} \cdot\left\{\boldsymbol{\nabla} n_{p}(\mathbf{r}, t)-\left(q / K_{B} T\right) n_{p}(\mathbf{r}, t) \mathbf{E}(\mathbf{r}, t)\right\},
$$

$$
\frac{\partial n_{m}(\mathbf{r}, t)}{\partial t}=D_{m} \boldsymbol{\nabla} \cdot\left\{\boldsymbol{\nabla} n_{m}(\mathbf{r}, t)+\left(q / K_{B} T\right) n_{m}(\mathbf{r}, t) \mathbf{E}(\mathbf{r}, t)\right\} .
$$

In Eq. (8) we have taken into account that $\mu_{p} / D_{p}=\mu_{m} / D_{m}$ $=q / K_{B} T$, where $K_{B} T$ is the thermal energy, known as relation of Einstein-Smoluchowski [26]. The electric field appearing in Eq. (8) has to be determined by solving the equation of Poisson, relating the actual electric field with the charge distribution [1]

$$
\boldsymbol{\nabla} \cdot \mathbf{E}(\mathbf{r}, t)=(q / \varepsilon)\left\{n_{p}(\mathbf{r}, t)-n_{m}(\mathbf{r}, t)\right\},
$$

where $\varepsilon$ is the dielectric constant of the medium in which the ions are dispersed.

If the actual electric field in the sample is so small that the variations in the bulk density of positive and negative ions with respect to its value of equilibrium, $N, \delta n_{p}(\mathbf{r}, t)$ $=n_{p}(\mathbf{r}, t)-N$ and $\delta n_{m}(\mathbf{r}, t)=n_{m}(\mathbf{r}, t)-N$ are such that $\delta n_{p}(\mathbf{r}, t) \ll N$ and $\delta n_{m}(\mathbf{r}, t) \ll N$, the fundamental equations of the problem, Eq. (8) reads as

$$
\begin{gathered}
\frac{\partial n_{p}(\mathbf{r}, t)}{\partial t}=D_{p} \boldsymbol{\nabla} \cdot\left\{\boldsymbol{\nabla} n_{p}(\mathbf{r}, t)-\left(q / K_{B} T\right) N \mathbf{E}(\mathbf{r}, t)\right\}, \\
\frac{\partial n_{m}(\mathbf{r}, t)}{\partial t}=D_{m} \boldsymbol{\nabla} \cdot\left\{\boldsymbol{\nabla} n_{m}(\mathbf{r}, t)+\left(q / K_{B} T\right) N \mathbf{E}(\mathbf{r}, t)\right\} .
\end{gathered}
$$

In this situation, the equations describing the system are linear. Let us consider now the case where the sample is a slab of thickness $d$, limited by two perfectly blocking electrodes of surface area $S$. The $z$ axis of the Cartesian reference frame is normal to the limiting surfaces, coinciding with the electrodes, situated at $z= \pm d / 2$. In this framework, the equation describing the system under investigation, in the linear limit, are

$$
\begin{aligned}
& \frac{\partial n_{p}(\mathbf{r}, t)}{\partial t}=D_{p}\left\{\frac{\partial^{2} n_{p}(z, t)}{\partial z^{2}}+\frac{q N}{K_{B} T} \frac{\partial^{2} V(z, t)}{\partial z^{2}}\right\}, \\
& \frac{\partial n_{m}(\mathbf{r}, t)}{\partial t}=D_{m}\left\{\frac{\partial^{2} n_{m}(z, t)}{\partial z^{2}}-\frac{q N}{K_{B} T} \frac{\partial^{2} V(z, t)}{\partial z^{2}}\right\},
\end{aligned}
$$

and

$$
\frac{\partial^{2} V(z, t)}{\partial z^{2}}=-\frac{q}{\varepsilon}\left\{n_{p}(z, t)-n_{m}(z, t)\right\},
$$

where $V(z, t)$ is the electrical potential related to the electric field by $E(z, t)=-\partial V(z, t) / \partial z$. If the voltage applied to the sample by means of an external power supply is sinusoidal of amplitude $V_{0}$ and angular frequency $\omega$, Eqs. (11) and (12) have to be solved with the boundary conditions

$$
\begin{aligned}
& j_{p}( \pm d / 2, t)=0, \quad j_{m}( \pm d / 2, t)=0, \\
& \quad \text { and } \quad V( \pm d / 2, t)= \pm\left(V_{0} / 2\right) \cdot \exp (i \omega t),
\end{aligned}
$$

related to the hypotheses that the electrodes are perfectly blocking and to the presence of the external power supply. Due to the linearity of the equation of the problem, $\delta n_{p}(z, t)=n_{p}(z, t)-N, \delta n_{m}(z, t)=n_{m}(z, t)-N$, and $V(z, t)$ are 
also simple sinusoidal functions of $t$. By putting

$$
\begin{gathered}
\left\{\delta n_{p}(z, t), \delta n_{m}(z, t)\right\}=\left\{\eta_{p}(z), \eta_{m}(z)\right\} \exp (i \omega t) \\
\text { and } \quad V(z, t)=\nu(z) \exp (i \omega t),
\end{gathered}
$$

it is possible to rewrite Eqs. (11) and (12) as

$$
\begin{gathered}
i \omega \eta_{p}(z)=D_{p}\left\{\eta_{p}^{\prime \prime}(z)+\left(q N / K_{B} T\right) \nu^{\prime \prime}(z)\right\}, \\
i \omega \eta_{m}(z)=D_{m}\left\{\eta_{m}^{\prime \prime}(z)-\left(q N / K_{B} T\right) \nu^{\prime \prime}(z)\right\}, \\
\nu^{\prime \prime}(z)=-(q / \varepsilon)\left\{\eta_{p}(z)-\eta_{m}(z)\right\},
\end{gathered}
$$

where the prime means derivative with respect to $z, X^{\prime}(z)$ $=d X(z) / d z$. In this case it is possible to evaluate the electrical impedance of the cell, $Z[1-3]$. When the diffusion coefficients of the positive and negative ions are identical, $D_{p}$ $=D_{m}=D, Z$ can be written in the form $[4,27]$

$$
Z=-i \frac{2}{\omega \varepsilon \beta^{2} S}\left\{\frac{1}{\lambda^{2} \beta} \tanh \left(\frac{\beta d}{2}\right)+i \frac{\omega d}{2 D}\right\},
$$

where $\lambda^{2}=\varepsilon K_{B} T /\left(2 N q^{2}\right)$ is the length of Debye, and

$$
\beta=\frac{1}{\lambda} \sqrt{1+i \frac{\omega}{D} \lambda^{2}}
$$

is a complex wave vector. From Eq. (16) it is possible to determine the real, $R$, and imaginary, $X$, parts of $Z$, known as the resistance and reactance of the cell. According to Eq. (16), the $R$ presents a large plateau, ending at the frequency of Debye, defined by $\omega_{D}=D / \lambda^{2}$. For $\omega \leq \omega_{D}, \quad R$ $\sim\left(\lambda^{2} / \varepsilon D\right)(d / S)$, and for $\omega \geq \omega_{D}, R$ tends to zero as $\omega^{-2}$. From Eq. (16) it follows also that in the limit $\omega \rightarrow 0$, the effective capacitance, $C_{\mathrm{eq}}$ of the cell tends to $C_{\mathrm{eq}}$ $\rightarrow \varepsilon S /(2 \lambda)$. In the opposite limit, where $\omega \rightarrow \infty, C_{\mathrm{eq}} \rightarrow \varepsilon S / d$. At the frequency of Debye the real and imaginary parts of $\beta^{2}$, defined by Eq. (17), are equal.

\section{GENERALIZATION OF THE LAW OF FICK}

From Eq. (5) it follows that in the case of an unbounded domain, if the initial condition on the bulk distribution of the particles is of the type

$$
n(\mathbf{r}, 0)=\mathcal{N} \delta\left(\mathbf{r}-\mathbf{r}_{0}\right),
$$

where $\mathcal{N}$ is the number of particles and $\delta\left(\mathbf{r}-\mathbf{r}_{0}\right)$ is the function of Dirac centered around $\mathbf{r}_{0}$, the bulk density of particles is, for $t \geq 0$ given by

$$
n(\mathbf{r}, t)=\frac{\mathcal{N}}{(4 \pi D t)^{3 / 2}} \exp \left\{-\frac{\left(\mathbf{r}-\mathbf{r}_{0}\right)^{2}}{4 D t}\right\},
$$

that for $t \rightarrow 0$ is different from zero in all points of the domain. This means that the velocity of the bulk variation in density is infinite. Since this result follows from the integration of the diffusion equation, consequence of the law of Fick, one can conclude that the law of Fick is an approximation for the diffusion current. As is well known, if one faces the diffusion problem by means of the transport equation of Boltzmann, this absurd result is absent [28]. Based on physi- cal arguments, long ago Cattaneo [23] proposed to modify the law of Fick. The phenomenological derivation of the equation of Cattaneo in the case of diffusion is based on the assumption that the flux of particles $\mathbf{j}(\mathbf{r}, t)$ is given by the equation

$$
\mathbf{j}(\mathbf{r}, t)+\tau \frac{\partial \mathbf{j}(\mathbf{r}, t)}{\partial t}=-D \nabla n(\mathbf{r}, t)
$$

where $\tau$ is a positive parameter having the dimensions of time [29]. Equation (20) holds in the approximation that the system presents only one delay time, rather than a distribution of such times. We limit our analysis to this case. For $\tau$ $=0$ we recover the law of Fick. Equation (20) can be considered as an approximation of the functional relation

$$
\mathbf{j}(\mathbf{r}, t+\tau)=-D \nabla n(\mathbf{r}, t),
$$

when $\tau$ is a small parameter. From Eq. (20) we obtain

$$
\boldsymbol{\nabla} \cdot \mathbf{j}(\mathbf{r}, t)+\tau \boldsymbol{\nabla} \cdot\left(\frac{\partial \mathbf{j}(\mathbf{r}, t)}{\partial t}\right)=-D \boldsymbol{\nabla}^{2} n(\mathbf{r}, t),
$$

that by inverting the order of the derivatives in the second term can be rewritten as

$$
\boldsymbol{\nabla} \cdot \mathbf{j}(\mathbf{r}, t)+\tau \frac{\partial}{\partial t}[\boldsymbol{\nabla} \cdot \mathbf{j}(\mathbf{r}, t)]=-D \boldsymbol{\nabla}^{2} n(\mathbf{r}, t)
$$

By taking into account the equation of continuity, Eq. (2), from Eq. (23) we get

$$
\frac{\partial n(\mathbf{r}, t)}{\partial t}+\tau \frac{\partial^{2} n(\mathbf{r}, t)}{\partial t^{2}}=D \nabla^{2} n(\mathbf{r}, t),
$$

which is the generalization of the diffusion equation to the hyperbolic diffusion regime model [28,29]. From Eq. (24) the velocity of propagation of the time variation of the bulk density variation is finite and given by $v=\sqrt{D / \tau}$.

In the case in which the particles under investigations are ions the generalization of the law of Fick, Eq. (20) is

$$
\begin{gathered}
\mathbf{j}_{p}(\mathbf{r}, t)+\tau_{p} \frac{\partial \mathbf{j}_{p}(\mathbf{r}, t)}{\partial t}=-D_{p} \boldsymbol{\nabla} \cdot n_{p}(\mathbf{r}, t)+\mu_{p} n_{p}(\mathbf{r}, t) \mathbf{E}(\mathbf{r}, t), \\
\mathbf{j}_{m}(\mathbf{r}, t)+\tau_{m} \frac{\partial \mathbf{j}_{m}(\mathbf{r}, t)}{\partial t}=-D_{m} \boldsymbol{\nabla} \cdot n_{m}(\mathbf{r}, t)-\mu_{m} n_{m}(\mathbf{r}, t) \mathbf{E}(\mathbf{r}, t),
\end{gathered}
$$

for the positive and negative ions, respectively. In Eq. (25) $\tau_{p}$ and $\tau_{m}$ are the phenomenological parameters for the two type of ions. By multiplying Eq. (25) by $\boldsymbol{\nabla}$, inverting in the second terms the time derivative with the spatial derivatives, and taking into account the continuity equations we get

$$
\begin{aligned}
\frac{\partial n_{p}(\mathbf{r}, t)}{\partial t}+\tau_{p} \frac{\partial^{2} n_{p}(\mathbf{r}, t)}{\partial t^{2}}= & D_{p} \boldsymbol{\nabla} \cdot\left\{\boldsymbol{\nabla} n_{p}(\mathbf{r}, t)\right. \\
& \left.-\left(q / K_{B} T\right) n_{p}(\mathbf{r}, t) \mathbf{E}(\mathbf{r}, t)\right\},
\end{aligned}
$$




$$
\begin{aligned}
\frac{\partial n_{m}(\mathbf{r}, t)}{\partial t}+\tau_{m} \frac{\partial^{2} n_{m}(\mathbf{r}, t)}{\partial t^{2}}= & D_{m} \boldsymbol{\nabla} \cdot\left\{\boldsymbol{\nabla} n_{m}(\mathbf{r}, t)\right. \\
& \left.+\left(q / K_{B} T\right) n_{m}(\mathbf{r}, t) \mathbf{E}(\mathbf{r}, t)\right\},
\end{aligned}
$$

which are Eq. (8) in the approximation of Cattaneo. In the linear limit Eq. (26) becomes

$$
\begin{aligned}
\frac{\partial n_{p}(\mathbf{r}, t)}{\partial t}+\tau_{p} \frac{\partial^{2} n_{p}(\mathbf{r}, t)}{\partial t^{2}}= & D_{p} \boldsymbol{\nabla} \cdot\left\{\boldsymbol{\nabla} n_{p}(\mathbf{r}, t)\right. \\
& \left.-\left(q N / K_{B} T\right) \mathbf{E}(\mathbf{r}, t)\right\}, \\
\frac{\partial n_{m}(\mathbf{r}, t)}{\partial t}+\tau_{m} \frac{\partial^{2} n_{m}(\mathbf{r}, t)}{\partial t^{2}}= & D_{m} \boldsymbol{\nabla} \cdot\left\{\boldsymbol{\nabla} n_{m}(\mathbf{r}, t)\right. \\
& \left.+\left(q N / K_{B} T\right) \mathbf{E}(\mathbf{r}, t)\right\} .
\end{aligned}
$$

When the sample is in the shape of a slab the fundamental equations of the problem are

$$
\begin{gathered}
\frac{\partial n_{p}(\mathbf{r}, t)}{\partial t}+\tau_{p} \frac{\partial^{2} n_{p}(\mathbf{r}, t)}{\partial t^{2}}=D_{p}\left\{\frac{\partial^{2} n_{p}(z, t)}{\partial z^{2}}+\frac{q N}{K_{B} T} \frac{\partial^{2} V(z, t)}{\partial z^{2}}\right\}, \\
\frac{\partial n_{m}(\mathbf{r}, t)}{\partial t}+\tau_{m} \frac{\partial^{2} n_{m}(\mathbf{r}, t)}{\partial t^{2}}=D_{m}\left\{\frac{\partial^{2} n_{m}(z, t)}{\partial z^{2}}-\frac{q N}{K_{B} T} \frac{\partial^{2} V(z, t)}{\partial z^{2}}\right\},
\end{gathered}
$$

and the equation of Poisson is still Eq. (12).

In the case where the electrodes are perfectly blocking, and the applied voltage is a simple harmonic function, as assumed above, the boundary conditions of the problem under investigation are Eq. (13). The solutions of the problem we are looking for are again of form (14). Consequently the $\eta_{p}(z), \eta_{m}(z)$, and $\nu(z)$ are solutions of the ordinary differential equations

$$
\begin{gathered}
i \omega \eta_{p}(z)-\tau_{p} \omega^{2} \eta_{p}(z)=D_{p}\left\{\eta_{p}^{\prime \prime}(z)+\left(q N / K_{B} T\right) \nu^{\prime \prime}(z)\right\}, \\
i \omega \eta_{m}(z)-\tau_{m} \omega^{2} \eta_{m}(z)=D_{m}\left\{\eta_{m}^{\prime \prime}(z)-\left(q N / K_{B} T\right) \nu^{\prime \prime}(z)\right\}, \\
\nu^{\prime \prime}(z)=-(q / \varepsilon)\left\{\eta_{p}(z)-\eta_{m}(z)\right\} .
\end{gathered}
$$

It is possible to rewrite Eq. (29) in the form

$$
\begin{gathered}
i \omega \eta_{p}(z)=\frac{D_{p}}{1+i \omega \tau_{p}}\left\{\eta_{p}^{\prime \prime}(z)+\left(q N / K_{B} T\right) \nu^{\prime \prime}(z)\right\}, \\
i \omega \eta_{m}(z)=\frac{D_{m}}{1+i \omega \tau_{m}}\left\{\eta_{m}^{\prime \prime}(z)-\left(q N / K_{B} T\right) \nu^{\prime \prime}(z)\right\}, \\
\nu^{\prime \prime}(z)=-(q / \varepsilon)\left\{\eta_{p}(z)-\eta_{m}(z)\right\} .
\end{gathered}
$$

By comparing Eq. (30) with Eq. (15) we see that the generalization of the law of Fick as suggested by Cattaneo is equivalent to the transformations

$$
D_{p} \rightarrow \mathcal{D}_{p}=\frac{D_{p}}{1+i \omega \tau_{p}} \quad \text { and } \quad D_{m} \rightarrow \mathcal{D}_{m}=\frac{D_{m}}{1+i \omega \tau_{m}}
$$

This means that in the framework of the extended thermodynamics, the diffusion coefficients are frequency dependent complex quantities. Consequently, the expression of the impedance of the cell, in the simple case where $D_{p}=D_{m}=D$ and $\tau_{p}=\tau_{m}=\tau$, is still given by Eqs. (16) and (17), but $D$ is now changed to $\mathcal{D}$ defined by

$$
\mathcal{D}=\frac{D}{1+i \omega \tau} .
$$

As it is clear from the analysis reported above, if $\tau \ll \tau_{D}$, where $\tau_{D}$ is the relaxation time related to the frequency of Debye, $\tau_{D}=1 / \omega_{D}=\lambda^{2} / D$, no modifications of the usual results are expected. On the contrary, for $\tau$ comparable with $\tau_{D}$ the trends of $R=R(\omega)$ and $X=X(\omega)$ differs from those obtained in the frame of the approximation of Fick. In the present case, the complex wave vector defined by Eq. (17) is given by

$$
\beta_{c}=\frac{1}{\lambda} \sqrt{1+i \omega \frac{\lambda^{2}}{\mathcal{D}}}
$$

that, by taking into account Eq. (32), can be rewritten as

$$
\beta_{c}=\frac{1}{\lambda} \sqrt{\left(1-\omega^{2} \frac{\lambda^{2} \tau}{D}\right)+i \omega \frac{\lambda^{2}}{D}} .
$$

The real part of $\beta_{c}^{2}$ vanishes for the circular frequency

$$
\omega_{r}=\sqrt{\frac{D}{\lambda^{2} \tau}} .
$$

Furthermore, the real and imaginary parts of $\beta_{c}^{2}$ are equal for

$$
\begin{gathered}
\omega_{1}=\frac{1}{2 \tau}\left(-1+\sqrt{1+4 \frac{D \tau}{\lambda^{2}}}\right), \\
\omega_{2}=\frac{1}{2 \tau}\left(1+\sqrt{1+4 \frac{D \tau}{\lambda^{2}}}\right) .
\end{gathered}
$$

A simple calculation shows that $\omega_{1}<\omega_{r}<\omega_{2}$. In the limit of $\tau \rightarrow 0, \omega_{r}$ diverges as $\tau^{-1 / 2}, \omega_{1}$ tends to $D / \lambda^{2}$, and $\omega_{2} \mathrm{di}-$ verges as $\tau^{-1}$.

\section{DISCUSSION}

From Eqs. (16) and (17), taking into account Eq. (32), we get that in the limit $\omega \rightarrow 0$ the impedance tends to

$$
Z=-i \frac{2 \lambda}{\varepsilon S} \frac{1}{\omega}+\frac{\lambda^{2} d}{\varepsilon D S}+\mathcal{O}\left(\omega^{2}\right)
$$

if $d \gg \lambda$. In this limit, the value of the electrical impedance is independent of $\tau$. In the opposite limit, where $\omega \rightarrow \infty$, we get 


$$
Z=-i \frac{d}{\varepsilon S} \frac{1}{\omega}+\frac{2 \lambda}{\varepsilon S}\left(\frac{D}{\lambda^{2} \tau}\right)^{3 / 2} \frac{1}{\omega^{4}}+\mathcal{O}\left(\omega^{-5}\right),
$$

whereas in the case of $\tau=0$ from Eqs. (16) and (17) in the same limit we obtain

$$
Z=-i \frac{d}{\varepsilon S} \frac{1}{\omega}+\frac{D}{\varepsilon \lambda^{2}} \frac{d}{S} \frac{1}{\omega^{2}}+\mathcal{O}\left(\omega^{-3}\right) .
$$

In the limit of $\omega \rightarrow \infty, \log R$ tends to 0 as $\omega^{-2}$ if $\tau=0$, whereas for $\tau \neq 0, \log R$ tends to 0 as $\omega^{-4}$. Note that, in the high-frequency region, if $\tau \neq 0$, the real part of the impedance, $R$, is independent of the thickness of the sample, whereas if $\tau=0, R$ is proportional to it. In the same limit $\log (-X)$ tends to 0 as $\omega^{-1}$ for all $\tau$. The change of slope of $\log R$ vs $\omega$, in the limit of large $\omega$ takes place at circular frequency $\omega_{c}=1 / \tau$. In fact, if $\omega \lambda^{2} / D \gg 1$ and $\omega^{2} \lambda^{2} \tau / D \ll 1$, which imply $\omega_{D} \leq \omega \leq \omega_{r}$, we have

$$
\beta_{c} \sim \frac{1}{\lambda} \sqrt{1+i \omega \frac{\lambda^{2}}{D}}
$$

and the effect of delay can be neglected. In this case $\log R$ depends on $\omega^{-2}$, as predicted by Eq. (39). On the contrary, if $\omega \lambda^{2} / D \gg 1$ and $\omega^{2} \lambda^{2} \tau / D \gg 1$, which imply $\omega \gg \omega_{r}$ defined by Eq. (35), the delay plays an important role, and $\log R$ depends on $\omega^{-4}$, in agreement with Eq. (38). The terms $\omega^{2} \lambda^{2} \tau / D$ and $\omega \lambda^{2} / D$ are comparable for $\omega=1 / \tau$, as stated above.

For the numerical simulation we assume that the dielectric constant of the insulating liquid is of the order of $\varepsilon=80 \cdot \varepsilon_{0}$, where $\varepsilon_{0}$ is the dielectric constant of the vacuum, the bulk density of ions is $N=4 \times 10^{20} \mathrm{~m}^{3}$, and the diffusion coefficient $D_{p}=D_{m}=D=8.2 \times 10^{-11} \mathrm{~m}^{2} / \mathrm{s}$, typical for hydrogels. In this case the length of Debye is $\lambda=3.7 \times 10^{-7} \mathrm{~m}$, and the circular relaxation frequency $\omega_{D}=D / \lambda^{2}=593 \mathrm{rad} / \mathrm{s}$. The geometrical parameters of the cell are supposed to be $d=1 \mathrm{~mm}$ and $S=2 \times 10^{-4} \mathrm{~m}^{2}$. We consider the cases where the frequency related to $\tau, \omega_{c}=1 / \tau$ are $\omega_{c 1}=2 \pi$ $\times 10^{2} \mathrm{rad} / \mathrm{s}, \quad \omega_{c 2}=2 \pi \times 10^{4} \mathrm{rad} / \mathrm{s}, \quad$ and $\quad \omega_{c 3}=2 \pi$ $\times 10^{6} \mathrm{rad} / \mathrm{s}$ that correspond to the delay times $\tau_{1}=1.6$ $\times 10^{-3} \mathrm{~s}, \tau_{2}=1.6 \times 10^{-5} \mathrm{~s}$, and $\tau_{3}=1.6 \times 10^{-7} \mathrm{~s}$. The case related to $\omega_{c 4}$ corresponds to the situation in which there is no delay between the current density and the gradient of concentration. For all considered cases $\omega_{r}>\omega_{D}$.

In order to investigate the influence of the parameter of Cattaneo on the impedance of an electrolytic cell we consider the admittance $Y=1 / Z=G+i B$, and compare the spectra of $G$ and $B$ for a few a values of the parameter $\tau$ entering into Eq. (20). In Fig. 1 we show the spectra of $G=G(\omega)$ and $B=B(\omega)$ for the considered values of $\omega_{c}$. As it follows from Fig. 1(a), according to the value of $\omega_{c}$, it appears a change in the frequency dependence of $\log G$. In the limit of $\omega \rightarrow \infty$ from Eq. (38) it follows that $G \propto \omega^{-2}$, in agreement with the numerical results reported in Fig. 1(a). The frequency at which the variation takes place coincides with $\omega_{c}$, as discussed above. From Fig. 1(b) it is evident that $\log B$ is, practically, independent of $\omega_{c}$.
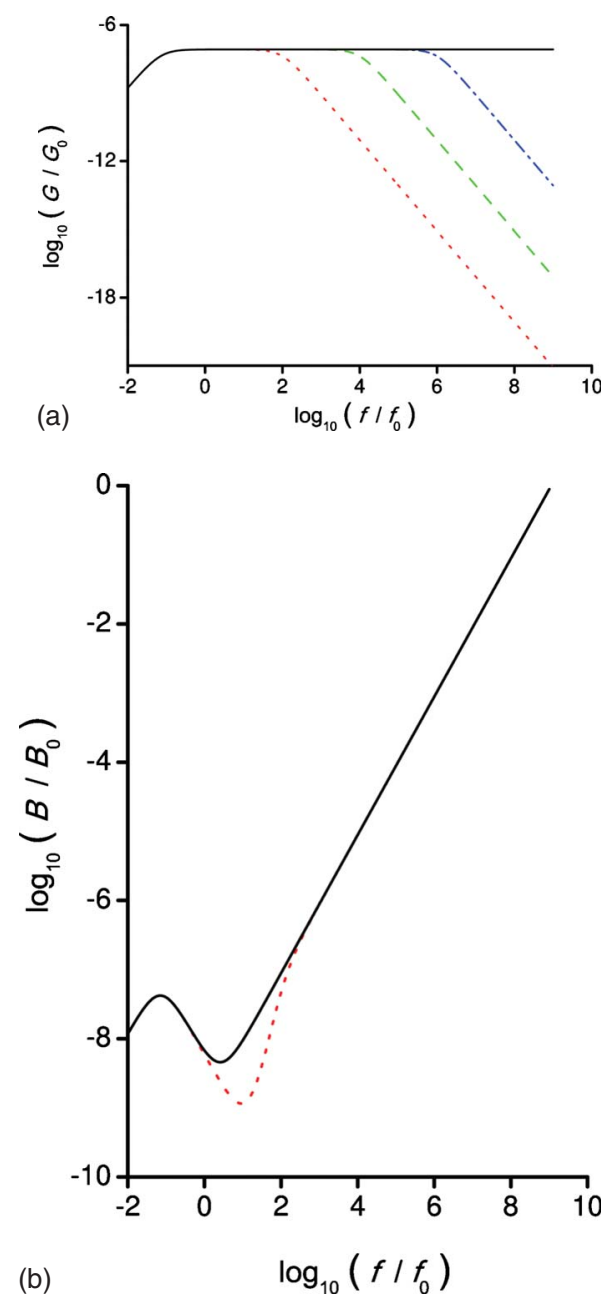

FIG. 1. (Color online) Frequency dependence of the (a) real part and (b) imaginary part of the admittance (in $\Omega^{-1}$ ) of an electrolytic cell for different values of the circular frequency $\omega_{c}=1 / \tau$, where $\tau$ is the characteristic delay time of the response of the system entering in the equation of Cattaneo. $G_{0}=1 \Omega^{-1}, B_{0}=1 \Omega^{-1}$, and $f_{0}$ $=1 \mathrm{~Hz}$. Dotted lines (red) $\omega_{c 1}=2 \pi \times 10^{2} \mathrm{rad} / \mathrm{s}$, dashed lines (green) $\omega_{c 2}=2 \pi \times 10^{4} \mathrm{rad} / \mathrm{s}$, and dotted-dashed lines (blue) $\omega_{c 3}$ $=2 \pi \times 10^{6} \mathrm{rad} / \mathrm{s}$. The continuous lines (black) correspond to the case in which there is not delay between the current density and the gradient of concentration $(\tau=0)$. With the numerical values used for $\omega_{c}$, the curves corresponding to $\omega_{c 2}$ and $\omega_{c 3}$ for the quantity $B$ coincide with the continuous black curve.

By means of the impedance of the cell it is possible to evaluate the real, $\epsilon^{\prime}$, and imaginary, $\epsilon^{\prime \prime}$, parts of the complex dielectric constant, $\epsilon=\epsilon^{\prime}-i \epsilon^{\prime \prime}$, of the cell defined by $Z$ $=-i /(\omega \mathcal{C})$ where the complex capacitance is given by $\mathcal{C}$ $=\epsilon(S / d)$. A simple calculation gives

$$
\epsilon^{\prime}=-\frac{1}{\omega} \frac{X}{R^{2}+X^{2}} \frac{d}{S} \text { and } \epsilon^{\prime \prime}=\frac{1}{\omega} \frac{R}{R^{2}+X^{2}} \frac{d}{S}
$$

By operating in a similar manner, it is possible to evaluate the real, $\sigma^{\prime}$, and imaginary, $\sigma^{\prime \prime}$, parts of the complex conductivity, $\sigma=\sigma^{\prime}+i \sigma^{\prime \prime}$, of the cell defined by $Z=\mathcal{R}$, where the complex resistance is defined by $\mathcal{R}=(1 / \sigma)(d / S)$. We get 

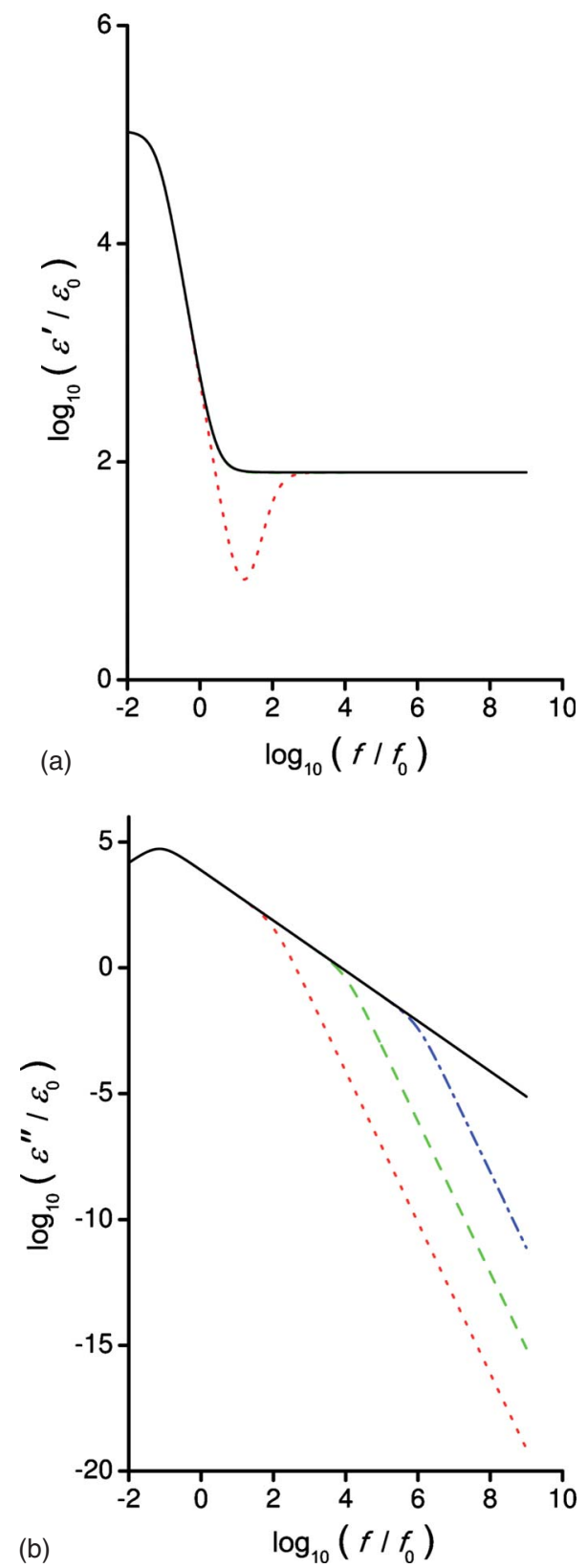

FIG. 2. (Color online) Frequency dependence of (a) $\epsilon^{\prime}$ and (b) $\epsilon^{\prime \prime}$ for different values of the circular frequency $\omega_{c}=1 / \tau$, where $\tau$ is the characteristic delay time of the response of the system entering in the equation of Cattaneo. $f_{0}=1 \mathrm{~Hz}$. Dotted lines (red) $\omega_{c 1}=2 \pi$ $\times 10^{2} \mathrm{rad} / \mathrm{s}$, dashed lines (green) $\omega_{c 2}=2 \pi \times 10^{4} \mathrm{rad} / \mathrm{s}$, and dotteddashed lines (blue) $\omega_{c 3}=2 \pi \times 10^{6} \mathrm{rad} / \mathrm{s}$. The continuous lines (black) correspond to the case in which there is not delay between the current density and the gradient of concentration $(\tau=0)$. With the numerical values used for $\omega_{c}$, the curves corresponding to $\omega_{c 2}$ and $\omega_{c 3}$ for the real part of the dielectric constant coincide with the continuous black curve.

$$
\sigma^{\prime}=\frac{R}{R^{2}+X^{2}} \frac{d}{S} \quad \text { and } \quad \sigma^{\prime \prime}=-\frac{X}{R^{2}+X^{2}} \frac{d}{S} .
$$

In Fig. 2 we show the spectra of (a) $\epsilon^{\prime}$ and (b) $\epsilon^{\prime \prime}$. From Fig. 2(a) it follows that small $\tau$ has a small influence on $\epsilon^{\prime}$. How-
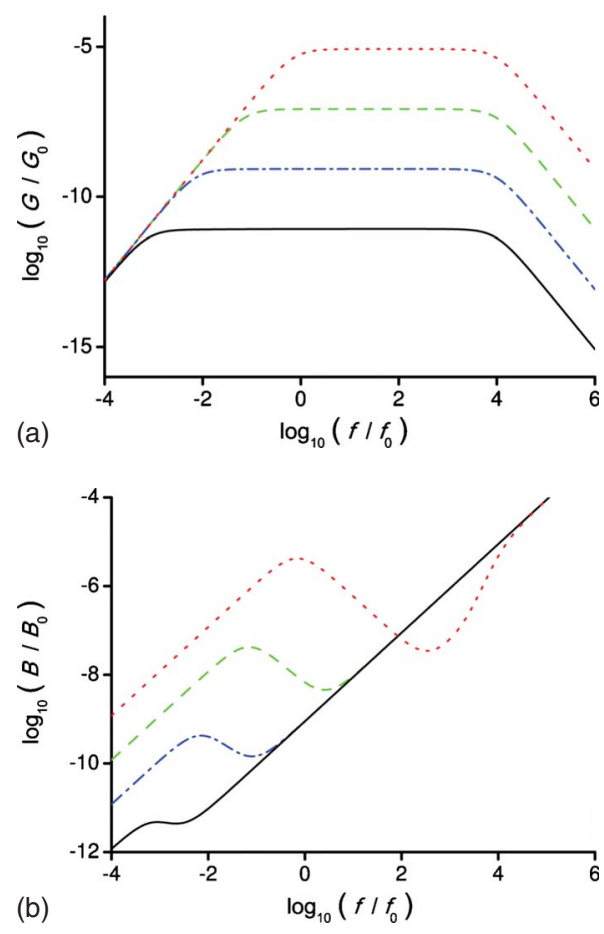

FIG. 3. (Color online) Frequency dependence of the (a) real part and (b) imaginary part of the admittance (in $\Omega^{-1}$ ) of an electrolytic cell for different values of the bulk density of ions $N$ for the circular frequency $\omega_{c}=2 \pi \times 10^{4} \mathrm{rad} / \mathrm{s} . G_{0}=1 \Omega^{-1}, B_{0}=1 \Omega^{-1}$, and $f_{0}$ $=1 \mathrm{~Hz}$. Dotted lines (red) $N=4 \times 10^{22} \mathrm{~m}^{-3}$, dashed lines (green) $N=4 \times 10^{20} \mathrm{~m}^{-3}$, and dotted-dashed lines (blue) $N=4 \times 10^{18} \mathrm{~m}^{-3}$, continuous lines (black) $N=4 \times 10^{16} \mathrm{~m}^{-3}$. By introducing the dimensionless quantity $M=d /(2 \lambda)$ that represents the number of length of Debye in a half cell, we have $M=13446, M=1344.6, M$ $=134.46$, and $M=13.44$, respectively.

ever, for the case corresponding to $\omega_{c 1}$, the real component of the dielectric constant has not a monotonic dependence on the frequency. On the contrary, the presence of a delay in the diffusion equation, changes the frequency dependence of $\epsilon^{\prime \prime}$, at the frequency $\omega_{c}=1 / \tau$, as it is evident for Fig. 2(b). In the limit of $\omega \rightarrow \infty, \epsilon^{\prime \prime} \propto \omega^{-3}$, as it follows from Eq. (38) and from the definition of $\varepsilon^{\prime \prime}$, Eq. (41), in agreement with the numerical results reported in Fig. 2(b).

In Fig. 3 we show the spectra of (a) $G$ and (b) $B$ for a different values of the bulk density of ions $N$. In Fig. 4 are reported the spectra of (a) $\epsilon^{\prime}$ and (b) $\epsilon^{\prime \prime}$ for the same values of $N$ shown in Fig. 3. As expected, increasing $N$ increases the frequency of Debye, which is related to $N$ by $\omega_{D}=D / \lambda^{2}$ $=2 N D q^{2} /\left(\varepsilon K_{B} T\right)$. Consequently, on increasing $N$ the correction due to the term of Cattaneo becomes more important, as it is evident from Figs. 3(b) and 4(a).

In the analysis presented above we have assumed $\omega_{c}$ $>\omega_{D}$, which implies $\tau$ smaller that the relaxation time of Debye, $\tau_{D}=\lambda^{2} / D$, which is the physical domain of interest. In the opposite case of $\omega_{r}<\omega_{D}$, the resistance of the cell, $R$, presents a maximum for a frequency close to $\omega_{r}$, and the reactance of the cell, $X$ passes from capacitive to pseudoinductive. In Fig. 5 we show the $R$, a, and $X, \mathrm{~b}$, for $N=4$ $\times 10^{20} \mathrm{~m}^{3}$ and $\omega_{c 1}=2 \pi \times 10^{1.5} \mathrm{rad} / \mathrm{s}, \omega_{c 2}=2 \pi \times 10^{2} \mathrm{rad} / \mathrm{s}$, $\omega_{c 3}=2 \pi \times 10^{2.5} \mathrm{rad} / \mathrm{s}$, and $\omega_{c 4}=2 \times 10^{4} \mathrm{rad} / \mathrm{s}$. In a Bode 

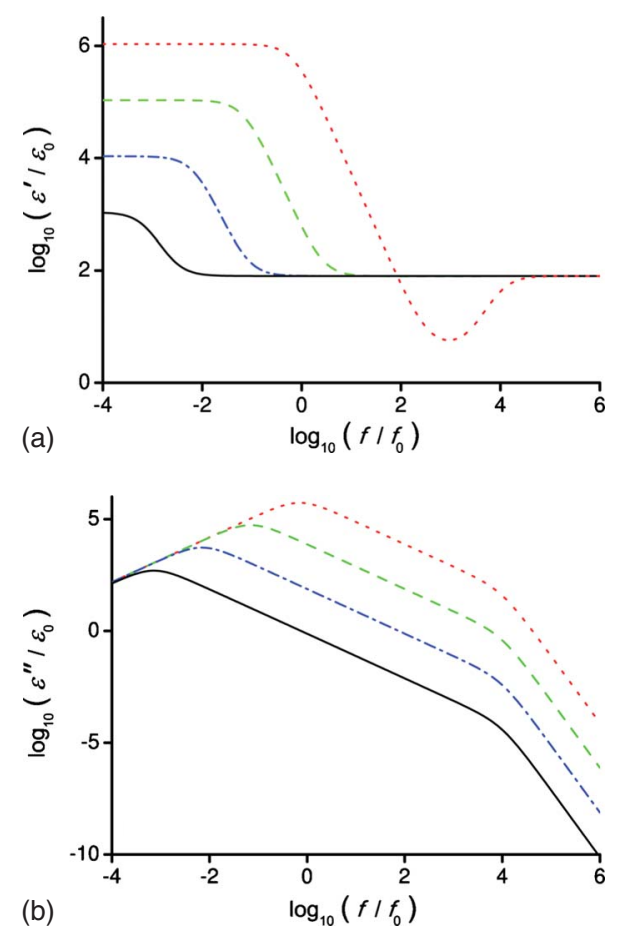

FIG. 4. (Color online) Frequency dependence of (a) $\epsilon^{\prime}$ and (b) $\epsilon^{\prime \prime}$ for different values of the bulk density of ions $N$ for the circular frequency $\omega_{c}=2 \pi \times 10^{4} \mathrm{rad} / \mathrm{s}$. $f_{0}=1 \mathrm{~Hz}$. Dotted lines (red) $N=4$ $\times 10^{22} \mathrm{~m}^{-3}$, dashed lines (green) $N=4 \times 10^{20} \mathrm{~m}^{-3}$, dotted-dashed lines (blue) $N=4 \times 10^{18} \mathrm{~m}^{-3}$, and continuous lines (black) $N=4$ $\times 10^{16} \mathrm{~m}^{-3}$.

representation, where is shown $|Z|=\sqrt{R^{2}+X^{2}}$ versus $f$ $=\omega /(2 \pi),|Z|$ presents a maximum for a frequency close to $\omega_{r}$. For the numerical values of the parameters used to draw Fig. 5, the frequency dependence of $M$ practically coincides with that of $R$ shown in Fig. 5(a). A similar effect has been reported by several authors [30-37], but at the present we have no clear indication that it is due to the nonvalidity of the law of Fick, or if it is just a phenomenon related to the electrodes. We hope that our paper can stimulate experimental researches to test the validity of the usual law of Fick for the ionic diffusion in insulating liquids by means of the impedance spectroscopy technique.

Although the pseudoinductive predictions of the hyperbolic response model are likely to be outside the realm of usual experimental results for liquids or solids involving completely blocking dispersive frequency response, could this model yield an explanation of the nearly endemic peak in observed at $\mathrm{THz}$ frequencies for various liquids and solids $[38,39]$. Such response has been often termed a "boson" peak, but it differs in shape for different materials and no general physical explanation for it seems available. It is worth noting that for the fragile glass former $3 \mathrm{KNO}_{3}-2 \mathrm{Ca}\left(\mathrm{NO}_{3}\right)_{2}(\mathrm{CKN})$, a molten salt, it appears likely that, in addition to a peak of $\epsilon^{\prime \prime}$ in the THz region, its $B$ response is also peaked in this region [40]. But, as shown by the results of Figs. 1 and 2, hyperbolic response leads to a peak in $B$ but not one in $\epsilon^{\prime \prime}$, vitiating any such possibility.
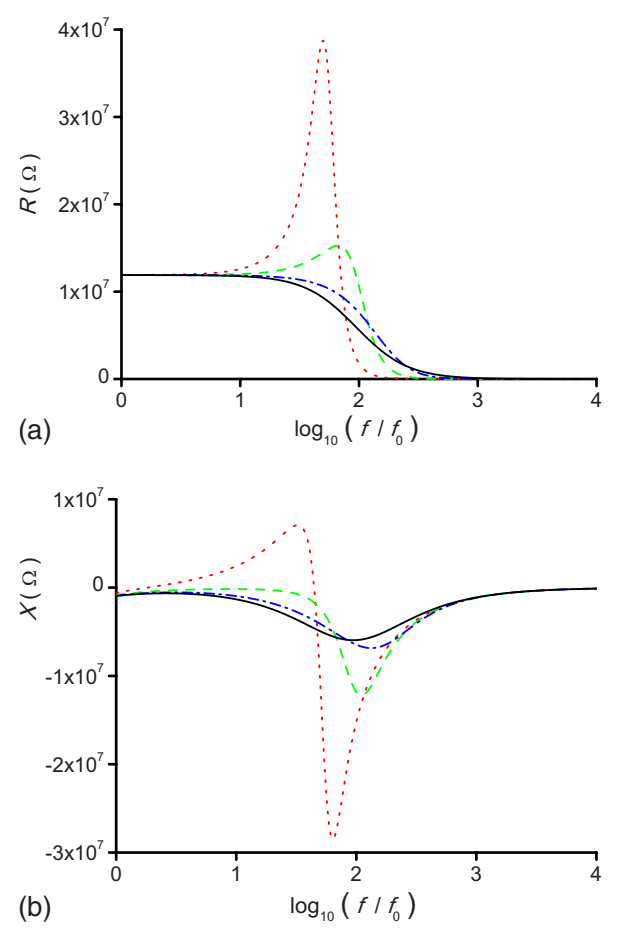

FIG. 5. (Color online) Frequency dependence of the (a) real part and (b) imaginary part of the impedance (in $\Omega$ ) of an electrolytic cell four different values of the circular frequency $\omega_{c}=1 / \tau$, where $\tau$ is the characteristic delay time of the response of the system entering in the equation of Cattaneo. $f_{0}=1 \mathrm{~Hz}$. Dotted lines (red) $\omega_{c 1}$ $=2 \pi \times 10^{1.5} \mathrm{rad} / \mathrm{s}$, dashed lines (green) $\omega_{c 2}=2 \pi \times 10^{2} \mathrm{rad} / \mathrm{s}$, and dotted-dashed lines (blue) $\omega_{c 3}=2 \pi \times 10^{2.5} \mathrm{rad} / \mathrm{s}$. The continuous lines (black) correspond to the case in which there is not delay between the current density and the gradient of concentration $(\tau$ $=0$ ).

\section{CONCLUSIONS}

We have extended the microscopic PNP theory relevant to the contribution of mobile ions to the electric properties of a dielectric medium incorporating a finite speed of response propagation. Our analysis has been limited to the case of small applied voltage, where the fundamental equations of the problem can be linearized, and to the case where the electrodes are perfectly blocking. In this framework we have shown that when the delay time of the response is comparable with the Debye relaxation time new trends for the real and imaginary parts of the electrical impedance of the cell are predicted and might be identified in experimental data especially by the presence of a decay of $\epsilon^{\prime \prime}$ with a highfrequency power-law exponent of -3 .

\section{ACKNOWLEDGMENTS}

This work has been partially supported by Regione Piemonte, Direzione Sanitá, Settore Promozione della Salute e Interventi di Prevenzione Individuale e Collettiva, in the frame of the project "Study of the electrical properties of the interface electrode-skin." Many thanks are due to M. Bazant and A. Scarfone for useful discussions 
[1] J. R. Macdonald, Phys. Rev. 92, 4 (1953).

[2] J. R. Macdonald, J. Chem. Phys. 58, 4982 (1973); J. Chem. Phys. 60, 343 (1974).

[3] J. R. Macdonald and D. R. Franceschetti, J. Chem. Phys. 68, 1614 (1978).

[4] J. R. Macdonald, J. Electroanal. Chem. 32, 317 (1971).

[5] J. R. Macdonald, J. Electrochem. Soc. 135, 2274 (1988), below Eq. A- 1 , in $\gamma_{j}$ replace $\theta_{j}^{2}$ by $\theta_{j}$, and on p. 2279 in $t_{1}$ replace $\psi$ by $\psi^{1 / 2}$.

[6] E. L. Cussler, Diffusion: Mass Transfer in Fluid System (Cambridge University Press, Cambridge, 1985).

[7] J. R. Macdonald and M. K. Brachman, J. Chem. Phys. 22, 1314 (1954).

[8] J. R. Macdonald, J. Chem. Phys. 30, 806 (1959).

[9] D. R. Franceschetti and J. R. Macdonald, J. Appl. Phys. 50, 291 (1979).

[10] D. R. Franceschetti, J. R. Macdonald, and R. Buck, J. Electrochem. Soc. 138, 1368 (1991).

[11] J. R. Macdonald, J. Chem. Phys. 61, 3977 (1974).

[12] J. R. Macdonald, Electrochim. Acta 37, 1007 (1992).

[13] R. Metzler and J. Klafer, Phys. Rep. 339, 1 (2000).

[14] R. Metzler and J. Klafer, J. Phys. A 37, R161 (2004).

[15] T. Kosztolowicz, K. Dworecki, and S. Mrowczynski, Phys. Rev. Lett. 94, 170602 (2005).

[16] T. Kosztolowicz, K. Dworecki, and S. Mrowczynski, Phys. Rev. E 71, 041105 (2005).

[17] T. Kosztolowicz and K. D. Lewandowska, J. Phys. A 42 , 055004 (2009).

[18] A. Compte and R. Metzler, J. Phys. A 30, 7277 (1997).

[19] C. Criado, V. Galan Montenegro, P. Velasquez, and J. R. Ramos Barrado, J. Electroanal. Chem. 488, 59 (2000).

[20] J. R. Ramos-Barrado, P. Galan Montenegro, and C. Criado Gambon, J. Chem. Phys. 105, 2813 (1996).
[21] K. D. Lewandoska and T. Kosztolowicz, Acta Phys. Pol. B 39, 1211 (2008).

[22] J. C. Maxwell, Philos. Trans. R. Soc. London 157, 49 (1867).

[23] G. Cattaneo, Atti Semin. Mat. Fis. Univ. Modena 3, 83 (1948).

[24] W. Kaminski, ASME J. Heat Transfer 112, 555 (1990).

[25] V. Smirnov, "Cours de Mathematiques Superieures," Tome II (MIR, Moscou, 1970).

[26] P. W. Atkins, Physical Chemistry (Oxford University Press, Oxford, 2004).

[27] G. Barbero and A. L. Alexe-Ionescu, Liq. Cryst. 32, 943 (2005).

[28] G. H. Weiss, Physica A 311, 381 (2002).

[29] D. D. Joseph and L. Preziosi, Rev. Mod. Phys. 61, 41 (1989); 62, 375 (1990).

[30] M. Bojinov, K. Salmi, and G. Sundholm, J. Electroanal. Chem. 347, 207 (1993).

[31] M. Bojinov, J. Electroanal. Chem. 405, 15 (1996).

[32] V. Alonzo, J. P. Diard, F. Berthier, and V. Seignole, J. Chim. Phys. 94, 1763 (1997).

[33] J. Lee, Ch. Eickes, M. Eiswirth, and G. Ertl, Electrochim. Acta 47, 501 (2001).

[34] J. Lee, Ch. Eickes, M. Eiswirth, and G. Ertl, Electrochim. Acta 47, 2297 (2002).

[35] S. Nakanishi, S.-I. Sakai, M. Hatou, Y. Mukouyama, and Y. Nakato, J. Phys. Chem. B 106, 2287 (2002).

[36] A. Sadkowski, J. Electroanal. Chem. 573, 241 (2004).

[37] A. Sadkowski, Solid State Ionics 176, 1987 (2005).

[38] P. Lunkenheimer, A. Pimenov, M. Dressel, B. Schiener, U. Schneider, and A. Loidl, Prog. Theor. Phys. 126, 123 (1997).

[39] P. Lunkenheimer and A. Loidl, J. Non-Cryst. Solids 352, 4556 (2006).

[40] J. R. Macdonald, J. Phys. Chem. B 113, 9175 (2009). 